\title{
A Smart Transmission Grid for Europe: Research Challenges in Developing Grid Enabling Technologies
}

\author{
Luigi Vanfretti, Member, IEEE, Dirk Van Hertem Senior Member, IEEE, \\ Lars Nordström, Member, IEEE, and Jan Ove Gjerde, Member, IEEE
}

\begin{abstract}
Smart grids have attracted significant attention lately, and one can even speak of a hype. However, much of the attention is paid to the distribution side and consumer interaction. Nevertheless, also at the transmission level important improvements can be achieved through farsighted and careful intelligent grid design and implementation. This paper identifies different research areas and their respective boundary interactions in order to enable a practical "Smart Grid" implementation in the European power system. Emphasis is placed on three essential aspects of the Smart Transmission Grid.

First, the necessary evolution of synchrophasor measurement technology is discussed, as well as the limitations towards and its full integration into power system operation and control. An important aspect to achieve this full integration is the necessity to test and integrate any proposed solution in an open and transparent environment. Secondly, the IT, data and communications paradigm is critically discussed. And lastly, the key questions that are open to the transmission system operators are discussed, specifically regarding the coordination within the pan-European power system and its security.

Going beyond the purely academic point-of-view, this paper specifically aims to bring a realistic approach towards research for the transmission network.
\end{abstract}

Index Terms-Smart Transmission Grid, Synchronized Phasor Measurement Units, WAMS, WACS, WAPS, Coordinated Control, Information Exchange, Quality of Service

\section{INTRODUCTION}

\section{A. Smarter grids, also in transmission}

Smart grids have attracted significant attention lately, even so that one can speak of a hype. The raising interest in "Smart Grids" has prompted an ever-increasing wave of discussion regarding a more disruptive introduction of information and communication technologies (ICT) to increase efficiency in electricity delivery and power network management. However, much of the attention is paid to the distribution side and the consumer interaction [1], [2]. Especially in Europe the smart transmission grid has remained in the background. Synchronized phasor measurement units (PMU), their supporting ICT infrastructures, and PMU data applications are a notable exception, although that they are less common in Europe than in the USA [3]. Nevertheless, at the transmission level there are also several important improvements that can be achieved

Invited Paper, Bringing Smart Grids to the Next Level Panel Session, Working Group on European Electricity Infrastructure, IEEE PES General Meeting 2011.

L. Vanfretti and D. Van Hertem are with the Electric Power Systems Division, School of Electrical Engineering, Royal Institute of Technology (KTH), Stockholm, Sweden. E-mail: luigiv@kth.se, dirk.vanhertem@ieee.org. Luigi Vanfretti is supported by the STandUP for Energy collaboration initiative and the KTH School of Electrical Engineering. Dirk Van Hertem is supported by the $\mathrm{EKC}^{2}$ competence center in electric power engineering

L. Nordström is with the Industrial Information and Control Systems Division, School of Electrical Engineering, Royal Institute of Technology (KTH), Stockholm, Sweden. E-mail: lars.nordstrom@ee.kth.se

J.O. Gjerde is with the Norwegian TSO Statnett SF, Oslo, Norway. E-mail: jan.gjerde@statnett.no through farsighted and careful intelligent grid design and implementation. These improvements are indispensable to operate future electric power systems that will be challenged by unpredicted uncertainties brought upon by a higher penetration of renewable and variable energy sources, limited investments in transmission assets, and an ever higher demand for a more secure supply of electric energy at the lowest possible cost.

Many of these improvements are not ready to be immediately implemented in the current system. There is a specific need for research on some key aspects of the smart transmission system which are deemed essential for the full development and utilization of the future grid. However, a significant portion of the ongoing research seems to be out of touch with reality. This problem arises from the fact that many theoretical approaches are not benchmarked in a lifelike simulation environment using realistic test systems, approaches that do not sufficiently take into account current installations, communication protocols, practices and operation. Also the non-technical and organizational (or regulatory) background is not sufficiently taken into account. In a nutshell, "artificial" research environments, while useful for discussing theoretical concepts, limit practical implementations and may be unfit to cater to measurements, data, and organizational aspects arising in the real power network. It becomes climacteric to realize that several limitations and boundaries conditions exist and must be taken into account in order to avoid oversimplified or overly optimistic solutions that are not applicable in reality.

In this article, the desired framework in which smart transmission grid research must operate is described, and some policy incentives are outlined. Section I-B lays out the research outlook as seen from current European policy, hence helping redefine the most consequential research directions. In Section II we discuss several considerations that need to be considered so that monitoring and control of Smart Transmission Grids (STGs) can be practically achieved through synchrophasor measurement technology. Next, ICT research aspects which are eminent for STG implementation are discussed in Section III, while Section IV the current panEuropean operation challenges and coordination aspects are explained. The paper concludes in Section V.

\section{B. Smart transmission research defined}

A definition of smart grids is given by the SmartGrids European Technology Platform [4]:

A Smart Grid is an electricity network that can intelligently integrate the actions of all users connected to it - generators, consumers and those that do both - in order to efficiently deliver sustainable, economic and secure electricity supplies.

The same organization identifies key research areas in its strategic research agenda [5], where the main transmission challenges are:

- New architectures and new tools 


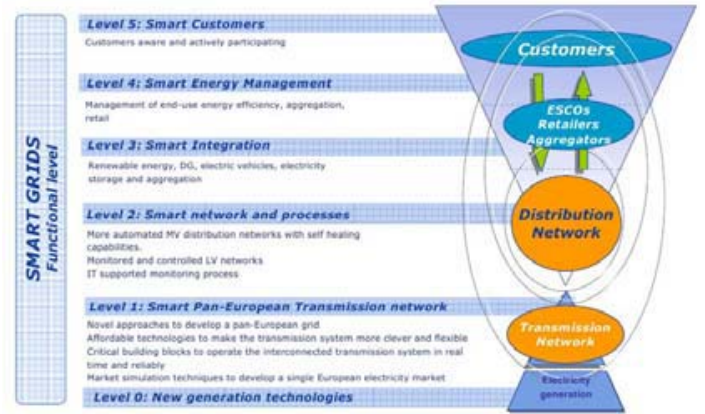

Fig. 1. Functional levels of the Smart Grid according to [10]

- Long distance energy supply

- European interoperability of smart grids

- Information and communication

The European Commission communicated in its "Blueprint for an integrated European energy network" [6] that it is necessary to have "rapid investments" in order to ensure "i) a competitive retail market, ii) a well-functioning energy services market which gives real choices for energy savings and efficiency, iii) the integration of renewable and distributed generation, and iv) to accommodate new types of demand, such as from electric vehicles". Next to these technical incentives, there is also a need for an update of the legal framework and to adapt legislations taking into account smart grids and smart meters [7], [8]. A higher transparency within the smart grids and an information platform is needed [6]. Much of this vision is also shared by the ERGEG (European Regulators Group of Electricity \& Gas) position paper on smart grids [9].

The European Electric Grids Initiative (EEGI) has indicated in its roadmap for RD\&D 2010-18 [10] a number challenges for the upcoming years. The document identifies 3 main action areas for the development of the future grid: i) the integration of new generation and consumption models, ii) a coordinated planning and operation of the pan-European grid, iii) new market models to maximize European welfare. The smart grids field is subdivided in different functional levels (see Fig. 1). Within the smart transmission grid level, the EEGI indicates four main research domains: the pan-European grid architecture, power technologies, network management and control and market rules. For each of these four areas, specific RD\&D activities are proposed. Observe that in Fig. 1 that the smart transmission network lies a foundational layer for the increased coordination between system operators (both TSO and DSO).

This paper focuses on the research challenges for transmission network management and control, and coordination aspects as indicated by EEGI. The IT, data and communication aspect are seen by EEGI as an integral part of the smart grid. However, in the authors opinion, several important research questions remain in the areas of syncrhonized phasor measurement techonologies, IT, data management and communications, which poses additional constraints to research and implementation.

Within this article, it is not the authors intention to give a comprehensive overview of all the outstanding issues and their binding research, but rather to cherry-pick some key challenges and potential pitfalls in the field of smart transmission system research.

\section{Monitoring And CONTROlling the STG}

A. Synchronized Phasor Measurement Technologies as building blocks of the STG

At the distribution side, most of the envisioned applications with regards to grid automation are technically seen feasible: the necessary components from ICT exist, and the required degree of automation needed to perform corrective and protective measures is possible with currently available power distribution assets. The on-going roll-out of "smart meters" allows an even more granular management of distribution networks and additional ventures into automated demand response [11]. Although there are still many challenges in this area, there exists many commercially available decision support computer and software systems, which can already exploit distribution assets [12]. Furthermore, much of the potential of additional smart meters lies in using the data from these meters to automatically adapt and react. While smart meters are becoming quite common, the feedback loop is far from commonplace.

The current approach for power system operations at the transmission level is to perform most of the monitoring and control actions within an Energy Management System (EMS), which makes use of a Supervisory Control and Data Acquisition (SCADA) system. There are many solutions available and currently used by transmission system companies and system operators. Although these systems are mature and dependable, it has not been until recently that wide-area features have been added to these systems. However, these features are not broadly adopted and have a reduced number of available phasor-data applications [13]. In addition, these systems where not developed to withstand the strain of managing the data volumes from the streaming of synchronized phasor measurements in an efficient manner [14]. Despite these limitations, there are initiatives in North America which have created specialized systems exploting phasor measurements with the aim of enabling new applications of PMU-data and increasing the utilization of synchrophasors in operations [15].

The new enabling technologies of so called wide-area monitoring systems are Phasor Measurement Units (PMUs) as the measurement device of choice, and their supporting infrastructure which is formed by communication networks and computer systems capable of handling PMU data and other information (usually called Phasor Data Concentrators (PDCs)). The set of PMUs and their enabling information and communication infrastructures is termed Synchrophasor Measurement Technology (SMT) [16]. With the rising number of synchrophasor installations around the world [17], a window of opportunity opens for stakeholders in the transmission system to exploit the time-stamped measurement data and higher resolution provided by PMUs. However, the number of applications available to transmission operators for exploiting 


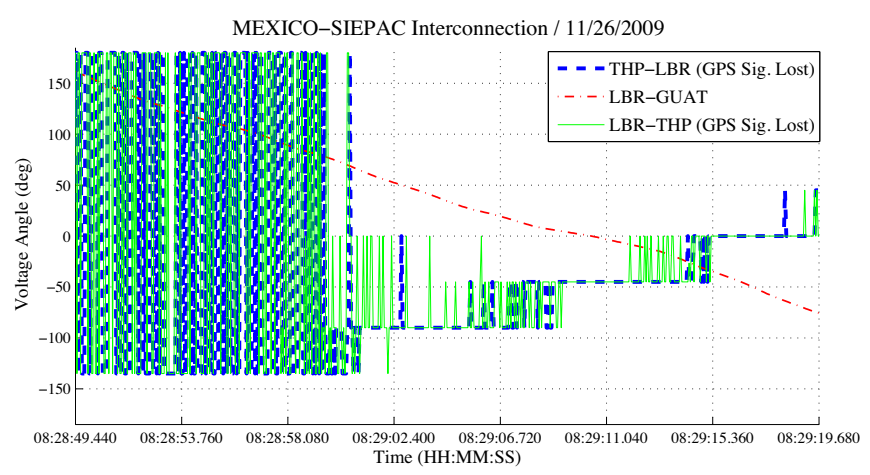

Fig. 2. Effect of GPS-signal loss in PMU data

these measurements seems to be insufficient to justify investments in SMT, and current projects dealing with installation of these devices are strongly subsidized [18]. Long-term development of these future power system technologies cannot rely on heavily subsided funding as its means to existence, and other economical drivers are needed. Hence, for the long term and sustained development of these technologies a different $\mathrm{R} \& \mathrm{D}$ approach is necessary.

\section{B. Motivation and causes}

There are several reasons for the lack of SMT-based applications and their limited adoption. These reasons emerge from the two different development approaches currently used: application development using real PMU data, and the simulation approach. From a researcher's stand point, obtaining real PMU data from transmission companies involves signing non-disclosure agreements which delays the start of research efforts, and more importantly, they may impose restrictions on the intellectual property of the derived works [19]. More importantly, when developing PMU-based applications, the PMU data itself is not sufficient: knowledge about the transmission system model parameters during the archived data time frame and other data (such as bus-bar level breaker status) are crucial for some applications [20], and may not be easy to obtain or interpret. Despite that the COMTRADE format has been selected for PMU data sharing in North America [21], due to regulations for post-mortem forensic analysis [22], this data format may not be the most convenient for application development and straightforward data analysis. ${ }^{1}$. Many applications require large records of phasor measurements (from 1 day to even weeks of archived data [24], and this from multiple PMU). Data availability and correct sharing mechanisms are not only an issue for academic researchers, but may also become important for application developers looking to extract features of data from massive data sets [25], otherwise, the industrial development and adoption of these applications can be further delayed. There are simply too many practical difficulties to develop PMU applications at a faster pace solely depending on measurements obtained from transmission utilities.

\footnotetext{
${ }^{1}$ Somehow, the power industry continues to overlook how other fields of research have dealt with massive amounts of data and have developed formats that allow to work and exchange numerical and graphical data efficiently R3]
}

On the other hand many academics have proposed applications of PMU data based solely on simulations using software commonly used in the power industry (which are mostly positive sequence-based (or phasor) simulations). While this approach is suited for some fundamental research, it might not be appropriate for actual implementation. This is because this approach does not take into account many of the challenges and characteristics of PMUs and the ICT systems. As a result, unreasonable assumptions of what the capabilities of these enabling technologies are made, often through an insufficient of the underlying technology limitations [26], although some of them have been acknowledged [27]. Simulating the use of remote data for control purposes is considerably easier than the actual implementation, where appropriate data filtering, transmission to a PDC, processing and transmission to the (remote) controller is needed. This requires several different stages where practical issues and possible delays occur. Without full consideration of these practical issues it may not be advisable to install these applications at a control center without going through a thorough testing process. An example of one of such practical issues is illustrated in Fig. 2, where the voltage phasor angle from PMUs installed at three different substations in the Mexican power system is shown. This figure shows the effect of GPS signal loss of in the THP-LBR and LBR-THP voltage angles. The trace of the LBR-GUAT angle shows no issues with the GPS-signal. Such issues with PMU measurements, and other similar ones [26], need to be taken into account while researching new methodologies so that these methods can be adequately employed by industry.

We realize that there is a dilemma on determining the appropriate ICT design specifications for each particular "application". The dilemma arises because not all the future applications enabling these STGs have been developed. In order to be developed correctly, they need "an" ICT infrastructure, which in turn needs application specifications for its design. A new approach for R\&D is necessary to flexibly evaluate different ICT paradigms at the same time that the power system operation and control strategies are being developed.

\section{How to move forward}

An STG is more than a grid that takes benefit of PMUs and requires ICT for this purpose. At a minimum, a STG should make use of this data in order to exploit all the available "observability" and "controllability" in a power system through closed-loop feedback control, and to coordinate system control with protection. As such it can behave as a "selfhealing" system, or at least utilizing the system more securely through increased awareness. To this extent, all measurement devices should be capable of producing synchronized and high-resolution time-stamped data that capture the dynamic behavior of the power system and can provide system observability. Controllability can be effectively provided by all those devices that can be in closed-loop control including conventional generation, Flexible AC Transmission Systems (FACTS), High Voltage Direct Current (HVDC), tap-changing and phase-shifting transformers, etc. 


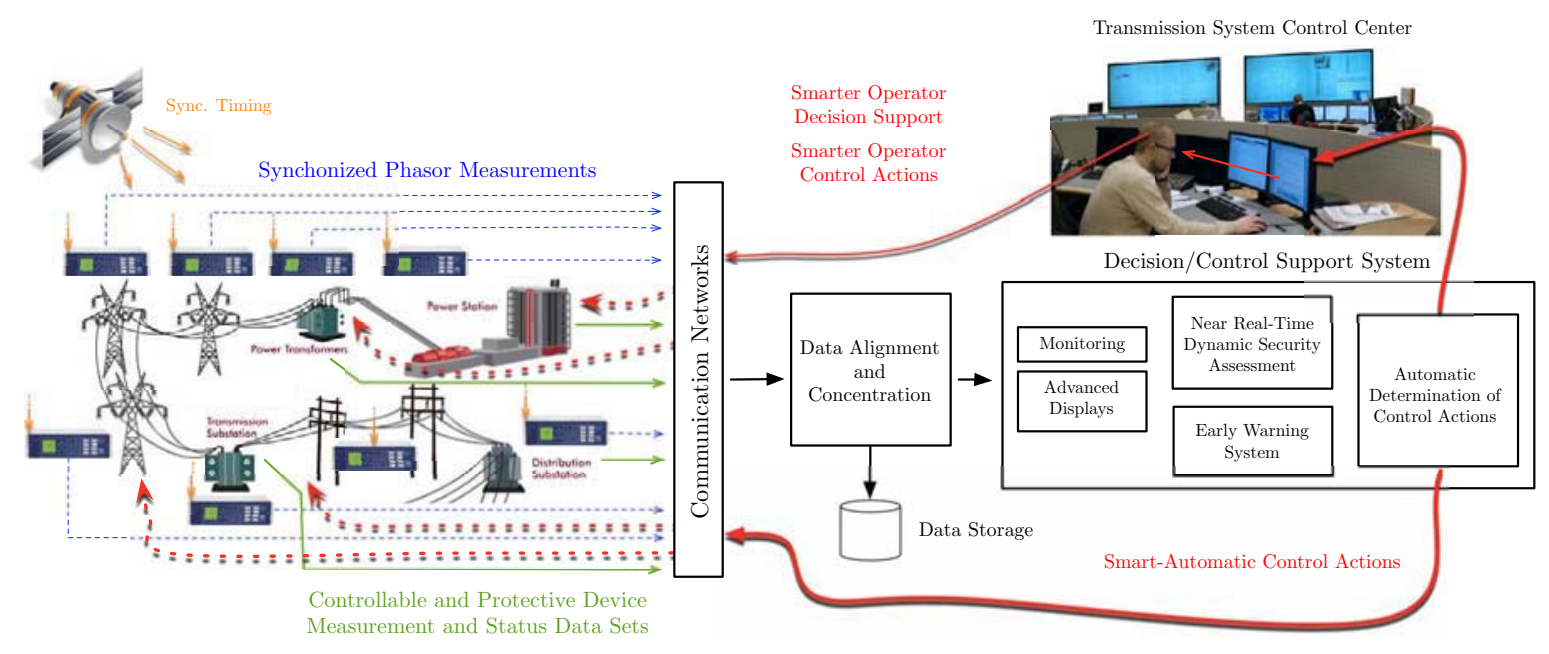

Fig. 3. A centralized model of a smart transmission grid

To accomplish these ambitions, STGs should contain more than the high-resolution measurements provided by PMUs. In Fig. 3, a conceptual diagram of a "centralized model" for a Smart Transmission Grid is shown. In such STG synchronized measurements are obtained at transmission substations through time synchronized measurements no only from PMUs but also from other envisioned highly accurate measurement systems retrieving data from controllable devices and protective device "information sets" (i.e. all available information from within a protective relay). This plethora of data is sent through communication networks, received and concentrated at a Decision and Control Support System that determines appropriate preventive, corrective and protective measures. This support system is the cornerstone for enabling STGs using synchrophasor data, it is here where the newly developed analysis techniques will produce "smarter" decisions allowing the power system to operate more securely, efficiently, and reliably. The decisions determined by this support system will then support operators at control centers to take "smarter operator control actions" or even device "smart-automatic control/protective actions". These actions are translated into feedback signals that are sent through communication networks to exploit the controllability and protection resources of the power system.

Note that although the diagram shown in Fig. 3 is a centralized model, there can be other more decentralized models for STGs. A "decentralized model" of a STG is shown in Fig. 4. Observe that in this paradigm we have divided the operation of the system into "focal area" systems with different operational functions (some of them might not include a focal area control center for example, implying that only other functions are taken there and thus a lower amount of data with perhaps lower Quality of Service (QoS) is needed) and a "wide-area" system. The data delivery is done through a publisher-subscriber model, such as GridStat [28], instead of a traditional star communication with round-robin polling model used in traditional EMS/SCADA systems, whose limitations have been acknowledged in [3]. Feasible approaches considered in [3], [28], [29] have great potential and should be further investigated.

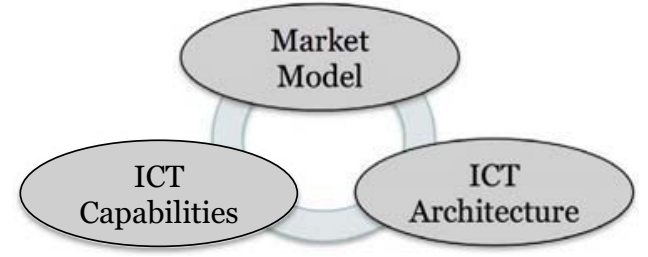

Fig. 5. ICT architecture and capabilities and their link with the market model

However, as mentioned before, the whole architecture of the system faces a dilemma as it will be determined by the requirements from different applications using PMU data, which in turn need the ICT infrastructure to be developed - in other words, how and for what purpose the measurement and other data be used will determine the most cost efficient system architecture. Yet, "an" architecture is needed to obtain data to develop the applications. To find the appropriate architecture that fits the needs of future power systems an appropriate platform for research is needed as well.

\section{ICT ASPECTS}

As is obvious from the preceding sections, implementation of the STG requires a supporting ICT infrastructure. As it has been indicated, there is a tendency to assume that the ICT systems will be adequate for all requirements from the power system [29]. Not only is this assumption incorrect, careless reliance on cheap and abundant ICT systems may also lead to over specified systems with undesirable CAPEX and OPEX expenditure. Just as the development of the power system requires proper methods for optimal design and operation, such methods should be employed for the ICT infrastructure supporting it. However, methods that cater for the interdependence of the power and ICT infrastructures are not commonly available, and this creates an overarching research challenge. In this section we present some challenges within the field requiring further scientific work.

\section{A. Market model implications}

Development of operational procedures and supporting ICT systems that enable the STG will be directed by the market 


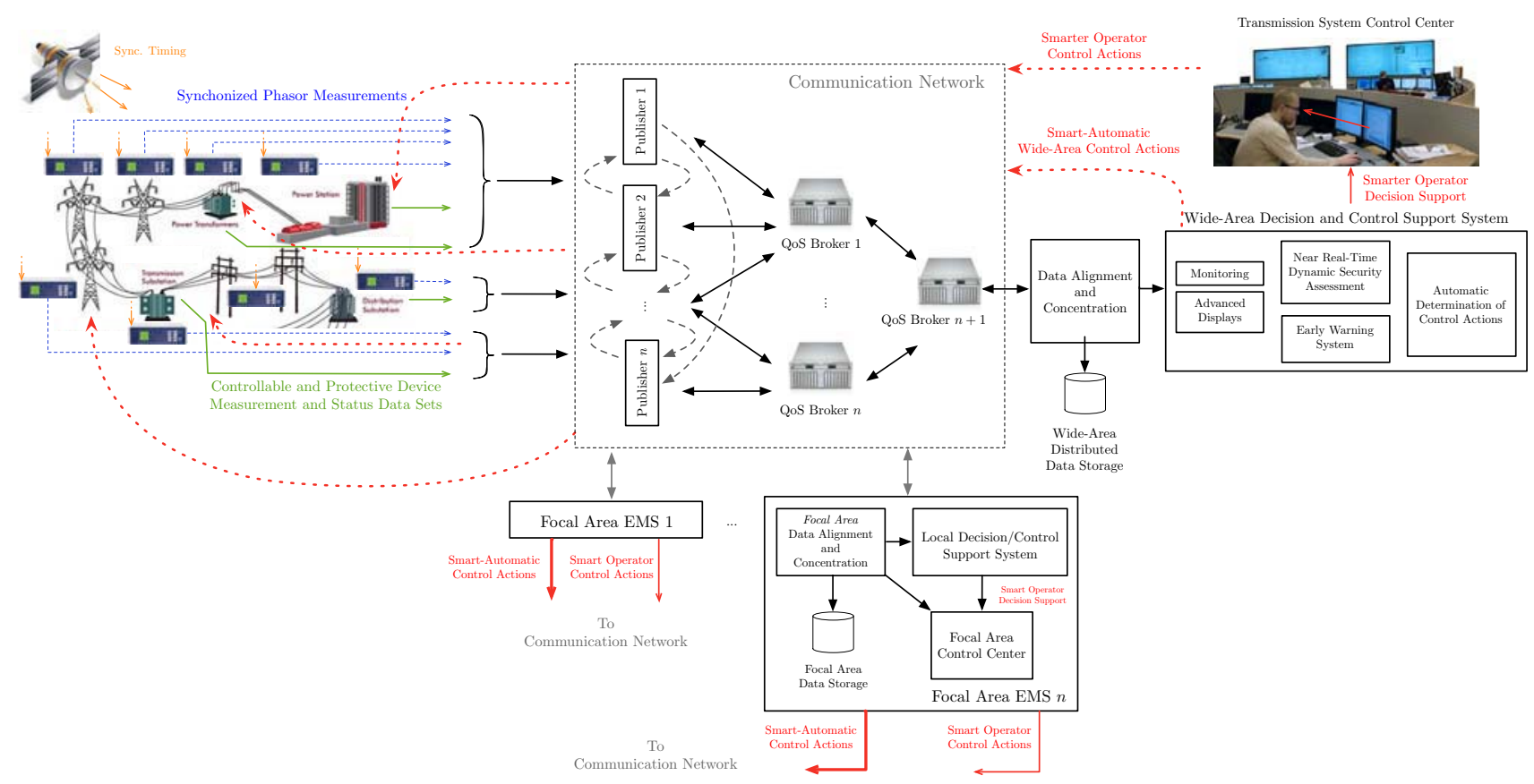

Fig. 4. A "decentralized model" of an STG

models, including regulation, that dictate the roles and responsibilities on the market.

However, the opposite is equally true, the possible architectures and the inherent ICT capabilities to some extent limit the possible market models. Consider for example minute level measurement and settlement on the power market. This is theory not different from hourly settlement, but the ICT systems needed clearly pose problems to implementing such a solution. Another more nuanced example is the storage of meter values and the implications this has on personal integrity, again illustrating how ICT aspects limits possible market solutions, see for instance [8]. One attractive approach to understanding this interaction between market and ICT system is model-based analysis using either multi-objective optimization or simulations using Multi-agent systems. Examples of such approaches can be found in [30] and [31]. To create generalisable results, such models must be open to modifications to represent different market models.

\section{B. ICT Impact on Observability and Controllability}

In general, the impact of the capabilities of the ICT systems on controllability and observability of the STG is poorly understood. Obviously, in general terms, ICT adds tremendously to the controllability and observability of power systems. However, given the increased interdependence of the ICT and power systems, the exact relation between critical ICT components and the power system needs improved methods for analysis. Within the critical infrastructure community this aspect has received considerable attention, see for instance [32] [33]. Understanding this relation requires formalized analysis of ICT components, and their relation to critical power system functions such as control, automation and protection. This later aspect is not the least important in the context of Cybersecurity, where unauthorized access to the control systems could potentially endanger the stability of the power system, see for example [34].

\section{Optimal information exchange}

Information exchange comes with a cost, both in direct terms with regards to bandwidth and database servers, but more importantly in terms of human and machine processing. An example of an extensive information exchange mechanism is the The Day Ahead Congestion Forecasting (DACF) [35] process being implemented in Europe. DACF involves exchange of power system models and estimates in order to create system wide models used for congestion management. The data volumes involved are extensive, and the resulting models are very detailed including time series of forecasted and estimated load flows. The development of the DACF process is clearly viable and necessary to enable optimal operation of the European grid. For processes with stricter time requirements than DACF; careful analysis of communication needs is critical to avoid over-specified systems with high investment and operational costs. This requires formalised methods for analysis of data volumes with relation to performance and quality requirements based on the actual power system control or planning needs.

\section{Agent based Distributed Control}

The unbundling of the power industry and introduction of new actors, such as aggregators create an environment in which decision-making is distributed. Such distributed systems require some form of coordination and and collaboration mechanism. One such promising approach towards collaboration is presented in the SEESGEN-ICT project where the concept of Service Level Agreements are used to control the interaction of different actors in the power system [36]. Such distributed ICT architectures are adaptable to changing 
requirements and contexts, however, this flexibility comes with a cost of increased traffic volumes, potential security threats as well as coordination and scalability challenges. This area requires extensive work to create foundations for robust multiagent architectures before these can be put into operation. For a good review, please see [37].

\section{E. Real time performance in WAMC}

An ICT system that spans a single TSOs control area seldom experiences performance problems when raw data transfer rates are considered. The abundance of optical fibers allows easy fast transportation of data from point $\mathrm{A}$ to $\mathrm{B}$. However, this fact is misleading, since end-to-end delay and throughput - the defining parameters within performance are effected by so many other factors than merely raw data transportation. That performance is a critical issue is evidenced in a number of studies related to WAMC [29] [38]. This method acknowledges the impact of the ICT performance on the power system, but does little towards understanding how this impact can be reduced or what trade-offs in latency reduction versus cost that is optimal. Additionally, the latency and throughput limitations faced in more advanced communications architectures employing layers of abstraction are still not considered [27]. More thorough studies on the impact various ICT architectures have on the possible power system control paradigms should be performed.

\section{F. Summary}

The preceding very brief review of ICT research challenges related to development of the Smart transmission Grid is intended to highlight the fact, that over-confidence in ICT solutions may lead to sub-optimal deployment of the STG. Cost efficient deployment of the STG includes optimised use of ICT, which in turn requires a more formal and knowledge based approach to the use of ICT.

\section{INTERNATIONAL OPERATION OF THE POWER SYSTEM}

\section{A. European framework}

In earlier days, before the mid nineties, the operation of the electric power system in Europe was rather straightforward, with vertically integrated companies that owned and controlled the entire value chain.

Through the process of liberalization, the European energy system became regulated and unbundled, with separated generation, transmission, distribution and retail.

The communication infrastructure is in many cases independent from the aforementioned companies, or belongs to a single one of them. In each country there is a regulator who sets the rules of the grid operation. In view of the smart grid, the regulator plays a vital player as he forms the main influence on grid rules, tariffs and has a strong voice in the investment process.

On the international level there are a number of organizations, associations and institutes that operate on a part of the transmission system related issues (examples are ENTSO-E, the association of transmission system operators in Europe, and ERGEG).

When looking at the interconnected European power system and its stakeholders, a complex patchwork of different entities with different tasks, objectives and non-overlapping geographic areas can be seen. When considering this international power system as the setting for the smart transmission grid, it is therefore essential to take these stakeholders into account

\section{B. System working up to its limits}

In the pre-liberalization era, the generators and grid were part of the same company that owned, maintained and operated the grid. The grid owner had the means to make the necessary investment decisions based on a coordinated planning (generation investments coordinated with necessary grid reinforcements). These generation investments were mostly traditional large generating units following the principle of economy of scale. The generation was usually located close to the load centers.

The system operator could control all aspects of the power system: generation for unit re-dispatch and managing the grid and its elements (line opening, capacitor switching, etc.) to control the system flows and avoid line overloads.

After being unbundled, the TSO still makes the investment decisions and manages the power system. However, he experiences several limitations in this respect. Generation investments are no longer coordinated with grid expansion, or rather, they are performed by independent organizations. The result is the shift of generators closer to the source of energy: e.g. the harbor for coal power plants.

The newly installed generation capacity is often also of a different type than it was before. Generation units from variable power energy sources are more commonplace due to the strive for more renewable energy generation and the emergence of other small scale generation such as CHP (combined heat and power). These devices are not only not predictable to a large extent, they are also uncontrollable by the operator. A more unpredictable power injection pattern will cause a higher uncertainty of the energy flows in the system. The increased market working has also led to a higher volatility of the energy flows, especially cross-border. At the same time there has been a lack of investments in the transmission system, specifically cross-border. The permitting process for generation is often considerably shorter than that of transmission, mainly due to projects that are postponed due to opposition from public, ecologists, etc.

The TSO can still control the grid to manage its flows, but re-dispatching generation has more difficult and costly.

As a result of the increase in variable energy flows, the limited grid investments and the reduced control options for the system operator, the grid is being operated closer to its limits, with potentially serious consequences for grid security. New and more advanced operation principles are needed in the system, through inter-zonal coordination and through enhanced security measures. 


\section{Coordination in the power system}

As the operation of the power system has become more inter-zonal, with higher and more variable power flows through the system, more coordination is needed. Especially because zones that are not directly taking part in energy transactions, are also affected by decisions made outside their zone. Currently, TSOs do coordinate the operation of the power system. There is an exchange in system data and operational information:

- Grid status (important scheduled outages)

- Day-ahead congestion forecasts are made

- Expected available transmission capacities are calculated

- Emergencies with possible effects beyond the local zone are communicated

However, the exchange happens on a very basic level. First of all, not all grid information is exchanged. One uses a "need to know" principle. An example is the load/generation data for DACF which are not shared independently, but rather as an aggregated quantity. Also information on the exact settings and status of devices (such as protection devices) is not always clearly communicated. One of the hurdles of this system is the exchange of data. There is also no common format that is used by the TSOs for these data exchanges, as most use different tools, possibly in-house developed.

Dynamic grid data is only rarely exchanged, and for this the data format is even more problematic as the different dynamic models used might differ significantly. Currently, no common data format exists, although that there are efforts in developing such a standard model (see CIM [39] and ODM [40]). However, significant improvements are needed in order to make them practical to use when many custom models are needed.

Not only the data sets that are used are different, also the tools and methodologies that are used differ amongst system operators. A good example is the N-1 rule, which is one of the fundamental security rules in the power system and well known to power system engineers. However, when going in detail it is clear that both the interpretation of " $\mathrm{N}$ " and "- 1 " can differ between organizations. Even within one TSO, the concept of N-1 can differ between the grid planning and the grid operation department.

Nevertheless, this system of "need to know" communication works reasonably well, with only a limited amount of grave events occurring due to mis-operation (e.g. Italy, August 2003; Germany, November 2006). Yet, potential problems remain:

- Unidentified loop flows occur

- The uncertainty in the system remains high

- The "limited amount" of grave events mentioned before could have been avoided

- Problems might be solvable on a local level at a high cost, but could have been solved at low expenses elsewhere

- There is no system-wide perspective

The existing coordination in the transmission system is currently sufficient to keep the lights on, but more is needed in order to make optimal use of the available resources. The current framework does not allow an integrated operation of the power system. Considering advanced operating principles without taking the current background of cooperation into account will lead to unrealistic results.

\section{Coordination of power flow controlling devices}

As a response to the lack of control means available to the system operator and to increase transmission capacity, several power flow controlling devices (PFC) have been installed in the European power system. Many of them are placed a meshed system of PFC.

A significant number of PFCs such as phase shifting transformers (PST) or HVDC lines are installed between different zones. Although PFC exhibit a strong influence on the flows through neighboring systems, these PFC are not operated in a coordinated way. As such, negative interactions between PFC, both during steady-state operation and through dynamic interactions may occur. This problem is especially important when the controllable devices are operated by different system operators. Therefore it is of substantial importance to deal with this issue when designing the controllers and during power system operation [41].

Most PFC are currently operated towards a certain objective which suits a single party. Coordination regarding PFC control is often limited to predefined rules and requires slow (often via telephone) interactions between the different participants. Coordination is limited for more than technical reasons: as the PFC is approved, installed and paid by a local entity, this entity will use that asset to its optimum, without considering the "bigger picture".

While there is significant potential in coordination of power flow coordinating devices, this potential is largely untapped at this moment. However, it is important to recognize the limitations in the system rather than searching for novel controller techniques that find no practical implementation.

\section{E. Secure operation of the pan-European power system}

The secure operation of the power system clearly has the highest priority for all system operators. However, there is currently no currently agreed upon methodology to accurately tackle the security question for the pan-European power system. It might even be needed to redefine the security principles to move beyond the classic N-1 approach in order to make optimal use of the existing power system while remaining within the operational bounds of the system.

Secondly, the European power system is the largest system engineered by men. At this moment no tools exist to adequately model and compute the entire system in a detailed and time efficient manner. New tools must be developed to enable the system operators to model and correctly control the pan-European network in the different time frames: from the millisecond range up to the operational planning range (days).

\section{CONCLUSiOn}

The challenges faced by the electric power industry are overwhelming, and its clear from the discussions in this 
paper that a "reality check" on current research practices is necessary; particularly if future power systems are going to hinge on the design of technologies and procedures emerging from the Smart Grid "hype". To this extent, if Smart Grids at the transmission level are to become a reality, there needs to be an alignment in the current research practices. This alignment should consider the climacteric boundary interactions between policies, the regulatory background, technology maturity and, socially-responsible and farsighted investment. Although we have not covered all possible aspects, we have highlighted the key-challenges and potential pitfalls in the field of STGs research.

\section{REFERENCES}

[1] R. Brown, "Impact of Smart Grid on distribution system design," in 2008 IEEE Power and Energy Society General Meeting - Conversion and Delivery of Electrical Energy in the 21st Century, 2008.

[2] G. T. Heydt, "The Next Generation of Power Distribution Systems," IEEE Transactions on Smart Grid, vol. 1, no. 3, pp. 225 -235, 2010

[3] A. Bose, "Smart Transmission Grid Applications and Their Supporting Infrastructure," IEEE Transactions on Smart Grid, vol. 1, no. 1, pp. 11 $-19,2010$.

[4] SmartGrids European Technology Platform, "Strategic deployment document for Europe's electricity networks of the future," SmartGrids ETP, Tech. Rep., 2010.

[5] — - "Strategic research agenda for Europe's electricity networks of the future," SmartGrids ETP, Tech. Rep., 2007.

[6] European Commission, "Energy infrastructure priorities for 2020 and beyond - a blueprint for an integrated european energy network," EC, Brussels, Tech. Rep. COM(2010) 677 final, nov 2010.

[7] J. Beyea, "The Smart Electricity Grid and Scientific Research," Science Magazine, vol. 328, no. 5981, pp. 979-980, 2010.

[8] Task Force Smart Grids Expert Group 2, "Regulatory Recommendations for Data Safety, Data Handling and Data Protection," EC, DG Energy, Direction for Security of Supply and Energy Markets, Tech. Rep., June 2010.

[9] European Regulators Group for Electricity \& Gas (ERGEG), "Position paper on smart grids - An ERGEG Public Consultation Paper," ERGEG, Tech. Rep. E09-EQS-30-04, dec 2009.

[10] European Electricity Grid Initiative (EEGI), "Roadmap 2010-18 and Detailed Implementation plan 2010-12," EEGI, Tech. Rep., may 2010.

[11] G. Thatikar, J. Mathieu, M. Piette, and S. Kiliccote, "Open Automated Demand Response Technologies for Dynamic Pricing and Smart Grid," in Grid-Interop Conference, Chicago, IL, 2010.

[12] C.G. Österbacka, "The Birth of MicroSCADA," 2003, available on-line: http://tinyurl.com/36c6myy.

[13] M. Zima, M. Larsson, P. Korba, C. Rehtanz, and G. Andersson, "Design aspects for wide-area monitoring and control systems," Proceedings of the IEEE, vol. 93, no. 5, pp. 980 -996, May 2005.

[14] J. Patterson, "Hadoop as the Platform for the Smartgrid at TVA," August 2010, available on-line: http://tinyurl.com/tva-hadoop.

[15] M. Patel (RAPIR Chair), "Real-Time Application of Synchrophasors Improving Reliability," North American Elecricity Reliability Corporation, Princeton, NJ, Tech. Rep., 2010.

[16] S. Chakrabarti, E. Kyriakides, T. Bi, D. Cai, and V. Terzija, "Measurements get together," IEEE Power and Energy Magazine,, vol. 7, no. 1, pp. $41-49,2009$.

[17] A. Phadke and R. de Moraes, "The wide world of wide-area measurement," IEEE Power and Energy Magazine, vol. 6, no. 5, pp. 52 -65, sep. 2008.

[18] U.S. Department of Energy, "Smart Grid Investment Grant Selectee Information," 2009. [Online]. Available: http://www.oe.energy.gov/recovery/1264.htm

[19] North American Synchrophasor Inititative (NASPI), "Naspi phasor data ndas," 2010. [Online]. Available: http://www.naspi.org/nda/nda.stm

[20] M. Kezunovic, "Intelligent Design: Substation DAta Integration for Enhanced Asset Management," IEEE Power and Energy Magazine,, vol. 8 , no. 6 , pp. $37-44,2010$.

[21] E. Allen, "Use of COMTRADE for Exchange of PMU Data," in NASPI Workgroup Meeting, Vancouver, Canada, June 2010.
[22] North American Electric Reliability Corporation (NERC), "Standard PRC-002-1 Define Regional Disturbance Monitoring and Reporting Requirements," 2006.

[23] T. H. Group, "What is HDF5?" [Online]. Available: http://www.hdfgroup.org/HDF5/whatishdf5.html

[24] L. Vanfretti, L. Dosiek, and et al, "Application of ambient analysis techniques for the estimation of electromechanical oscillations from measured PMU data in four different power systems," European Transactions on Electrical Power (Wiley), 2010, accepted for publication.

[25] P. Trachian, "Machine learning and windowed subsecond event detection on pmu data via hadoop and the openpdc," in IEEE Power and Energy Society General Meeting, 2010, pp. 1 -5.

[26] L. Vanfretti, J. H. Chow, S. Sarawgi, and B. B. Fardanesh, "A phasordata-based state estimator incorporating phase bias correction," IEEE Transactions on Power Systems, vol. To Appear, 2010.

[27] M. Chenine and L. Nordstrom, "Investigation of communication delays and data incompleteness in multi-pmu wide area monitoring and control systems," in International Conference on Electric Power and Energy Conversion Systems (EPECS), 2009, pp. 1 -6.

[28] H. Gjermundrod, D. Bakken, C. Hauser, and A. Bose, "GridStat: A Flexible QoS-Managed Data Dissemination Framework for the Power Grid," IEEE Transactions on Power Delivery, vol. 24, no. 1, pp. 136 $-143,2009$.

[29] D. Bakken, A. Bose, C. Hauser, E. O. S. III, D. Whitehead, and G. Zweigle, "Smart Generation and Transmission with Coherent, RealTime Data," Washington State University, Pullman, WA, Tech. Rep. TRGS-015, August 2010.

[30] G. Celli, F. Pilo, G. Pisano, and G. G. Soma, "Optimal Planning for Active Networks," in 16th Power Systems Computation Conference (PSCC), Glasgow, Scotland, 2008.

[31] S. Hussain and R. Gustavsson, "Coordinating Energy Based Business Models and Customer Empowerment in Future Smart Grids," in $7^{5 t}$ International ICST Conference on E-Energy, 2010.

[32] M. Viziteu, D. Georges, R. Caire, and N. Hadjsaid, "Criticality hierarchy procedure applied to software applications for electrical networks management," in 4th International Conference on Critical Infrastructures (CRIS), Apr. 2009, pp. $1-4$.

[33] G. D’Agostino, S. Bologna, V. Fioriti, E. Casalicchio, L. Brasca, E. Ciapessoni, and S. Buschi, "Methodologies for inter-dependency assessment," in 5th International Conference on Critical Infrastructure (CRIS), 2010, pp. $1-7$.

[34] T. Sommestad, M. Ekstedt, and L. Nordstrom, "Modeling Security of Power Communication Systems Using Defense Graphs and Influence Diagrams," IEEE Transactions on Power Delivery, vol. 24, no. 4, pp. $1801-1808,2009$.

[35] P. Panciatici and J. Vrsecky, "Day ahead Congestion Forecast for a Secure Operation of the European Transmission System," in 15th Power Systems Computation Conference (PSCC), Liège, Belgium, 2005.

[36] R. Gustavsson, H. Shahid, and B. Stå hl, "ICT For Data Management an Inter-Stakeholder Service Monitoring in Smart Grids," EU Project No.: 238868, SEESGEN-ICT, Tech. Rep., 2010.

[37] C. Rehtanz, Autonomous Systems and Intelligent Agents in Power System Control and Operation. Springer-Verlag, 2003.

[38] J. He, C. Lu, X. Jin, and P. Li, "Analysis of time delay effects on wide area damping control," in IEEE Asia Pacific Conference on Circuits and Systems (APCCAS), Dec. 2008, pp. $758-761$.

[39] E. Lambert, "Common information based on cim approach : Is it a dream or a reality? remaining challenges based on concrete experience," in IEEE Power and Energy Society General Meeting - Conversion and Delivery of Electrical Energy in the 21st Century, 2008, pp. $1-6$.

[40] F. Milano, M. Zhou, and G. Hou, "Open model for exchanging power system data," in IEEE Power Energy Society General Meeting,, 2009, pp. $1-7$.

[41] D. Van Hertem, R. Eriksson, L. Söder, and M. Ghandhari, "Coordination of multiple power flow controlling devices in transmission systems," in IET ACDC conference, London, October 2010. 